\title{
Asteroseismology of exoplanet host stars
}

\author{
Daniel Huber ${ }^{1,2,3}$ \\ ${ }^{1}$ Sydney Institute for Astronomy (SIfA), School of Physics, University of Sydney, NSW 2006, \\ Australia; email: daniel.huber@sydney.edu.au \\ ${ }^{2}$ SETI Institute, 189 Bernardo Avenue, Mountain View, CA 94043, USA \\ ${ }^{3}$ Stellar Astrophysics Centre, Department of Physics and Astronomy, Aarhus University, Ny \\ Munkegade 120, DK-8000 Aarhus C, Denmark
}

\begin{abstract}
Asteroseismology is among the most powerful observational tools to determine fundamental properties of stars. Space-based photometry has recently enabled the systematic detection of oscillations in exoplanet host stars, allowing a combination of asteroseismology with transit and radial-velocity measurements to characterize planetary systems. In this contribution I will review the key synergies between asteroseismology and exoplanet science such as the precise determination of radii and ages of exoplanet host stars, as well as applications of asteroseismology to measure spin-orbit inclinations in multiplanet systems and orbital eccentricities of small planets. Finally I will give a brief outlook on asteroseismic studies of exoplanet hosts with current and future space-based missions such as K2 and TESS.
\end{abstract}

Keywords. stars: oscillations, stars: late-type, planetary systems

\section{Introduction}

Exoplanet science has undergone a revolution in the past decade driven by high precision photometry from space-based missions such as CoRoT and Kepler. At the time of writing of this review Kepler has detected more than 4000 planet candidates (e.g. Mullally et al. 2015), with breakthrough discoveries including measurements of planet densities in multi-planet systems (e.g. Lissauer et al. 2011) and the detection of planets in the habitable zone (e.g. Quintana et al. 2014). In parallel, Kepler has enabled tremendous observational progress in asteroseismology, the study of stellar pulsations. In particular, the number of cool stars with detected oscillations driven by surface convection (solar-like oscillations) has increased by more than a order of magnitude, allowing unprecedented studies of their fundamental properties and interior structure (see Chaplin \& Miglio 2013, for a recent review).

The need for continuous high-precision time domain data (either in intensity or velocity) has enabled a fortuitous synergy between asteroseismology and exoplanet science since the data can be simultaneously used to detect exoplanets and study stellar oscillations (Figure 1). In this review I will discuss recent key synergies between asteroseismology and exoplanet science, and conclude with an outlook of what can expected from current and future space-based missions such as K2 and TESS (with a particular focus on asteroseismology of evolved host stars).

\section{Precise Characterization of Exoplanets}

Indirect detection methods such as transit and radial velocity surveys measure relative properties of exoplanets, hence requiring precise characterizations of their host stars. First asteroseismic studies of exoplanet host stars were performed using ground-based radial-velocity data (Bouchy et al. 2005; Bazot et al. 2005) or space-based photometry 


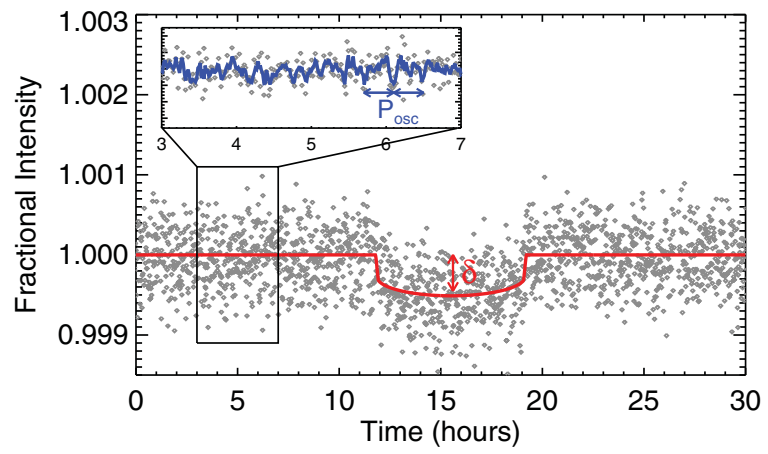

Figure 1. Kepler short-cadence data showing a single transit of Kepler-36c (Carter et al. 2012). The red solid line is the transit model, and the inset shows the oscillations of the host star. The transit depth $\delta$ yields the size of the planet relative to the star, and the oscillation periods $\left(P_{\text {osc }}\right)$ can be used to independently measure the size of the star.

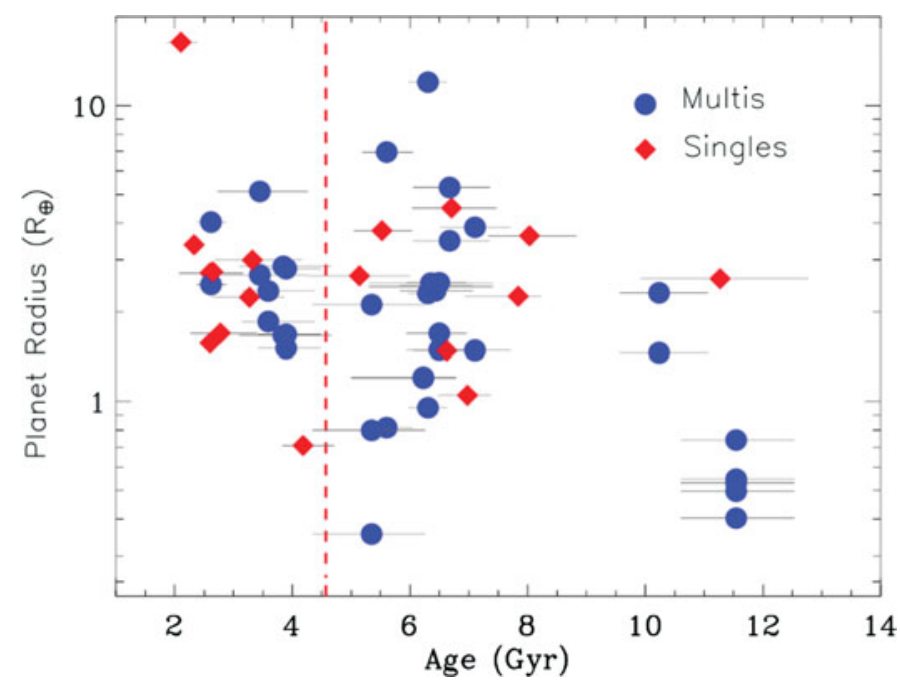

Figure 2. Planet radius versus host star age for a sample of 33 Kepler exoplanet host stars. The vertical dashed line marks the age of the Sun. Note that the bimodal distribution is mostly due to an asteroseismic detection bias towards more luminous stars (see text). Adapted from Silva Aguirre et al. (2015).

using the Hubble Space Telescope (Gilliland et al. 2011) and CoRoT (Ballot et al. 2011; Lebreton \& Goupil 2014). Following early asteroseismic studies supporting Kepler exoplanet detections (Christensen-Dalsgaard et al. 2010; Batalha et al. 2010; Howell et al. 2011), Huber et al. (2013a) presented the first systematic study for 77 Kepler host stars. In addition to the precise determination of a large number exoplanet radii, the results yielded systematic biases for surface gravities (and hence radii) for evolved stars based on high-resolution spectroscopy. The Kepler asteroseismic host star sample has since been used to calibrate more indirect methods to determine fundamental properties of exoplanet host stars such as high-resolution spectroscopy (Brewer et al. 2015) and stellar granulation (Bastien et al. 2013).

More recent studies have focused on not only measuring global asteroseismic quantities (which are sensitive to densities, masses and radii) but also systematic modeling of individual oscillation frequencies, which allows precise constraints on the stellar age 
(e.g. Metcalfe et al. 2012). Figure 1 shows the distribution of radii and ages of Kepler host stars from the first systematic age study by Silva Aguirre et al. (2015), based on individual frequencies measured by Davies et al. (2015). While the bimodal age distribution is mostly due to a detection bias towards evolved subgiants ( $\sim 6$ Gyrs) and more luminous $\mathrm{F}$ stars $(\sim 2-3 \mathrm{Gyrs})$, the analysis illustrated the exquisite precision with which ages can be determined $(\sim 14 \%)$. One of the most remarkable discoveries in the sample is Kepler-444, which consists of a $11.2 \pm 1.0$ Gyr year old $\mathrm{K}$ dwarf hosting five sub-Earth planets with orbital periods of less than 10 days (Campante et al. 2015). The system demonstrated that sub-Earth sized planets have existed for most of the history of our Universe, in line with earlier findings that the formation of small planets in independent of host star metallicity (Buchhave et al. 2012).

\section{Spin-Orbit Inclinations and Architectures of Exoplanet Systems}

In addition to fundamental properties, asteroseismology can be used to determine the line-of-sight inclination of the stellar rotation axis by measuring relative heights of rotationally split modes (Gizon \& Solanki 2003). For transiting exoplanets, a low stellar inclination automatically yields a misalignment of the orbital plane and the stellar equatorial plane (a high obliquity), while an inclination near 90 degrees implies that the star and the planets are likely (but not necessarily) aligned.

The long continuous time series by Kepler and CoRoT allowed first asteroseismic stellar inclination measurements for transiting exoplanet systems (Chaplin et al. 2013; Gizon et al. 2013; Van Eylen et al. 2014). An intriguing example is Kepler-56, a red giant hosting two transiting planets confirmed through transit-timing variations (Steffen et al. 2012). The Kepler-56 power spectrum revealed an inclination of $47 \pm 6$ degrees, demonstrating the first stellar spin-orbit misalignment in a multiplanet system (Huber et al. 2013b). Recent studies have since also determined the spin-axis inclination in systems for which the projected obliquity has been measured using the Rossiter-McLaughlin effect, which allows a determination of the 3D obliquity angle (Lund et al. 2014; Benomar et al. 2014).

Asteroseismic inclination measurements have played an increasingly important role for constraining formation theories for hot Jupiters. Figure 3 shows the projected obliquity or stellar inclination for exoplanet systems as a function of the relative tidal dissipation timescale, a proxy for how quickly a system can be realigned by tidal interactions if it was initially misaligned by the formation process (Albrecht et al. 2012). Hot Jupiter systems with intermediate dissipation timescale are frequently observed to have high obliquities (e.g. Winn et al. 2010), while coplanar multiplanet systems without hot Jupiters have have mostly low obliquities (e.g. Sanchis-Ojeda et al. 2013) despite long tidal dissipation timescales. This has been taken as evidence that the formation of hot Jupiters is related to dynamical interactions, rather than migrations through a protoplanetary disk.

The misalignment in the Kepler-56 system has yielded the first outlier in this trend. However, other seismic measurements yielded well aligned multiplanet systems, including the recent discovery of the Kepler-432 system which consists of a red giant with a transiting warm Jupiter and an outer giant planet detected through radial velocities (Quinn et al. 2015). Remarkably, Figure 3 shows that most constraints on orbital architectures for small multiplanet systems come from asteroseismology, since the technique is independent of planet size (unlike Rossiter-McLaughlin observations). Future asteroseismic inclination measurements will increase the sample and help to determine whether spin-orbit misalignments in multiplanet systems without hot Jupiters are common. 


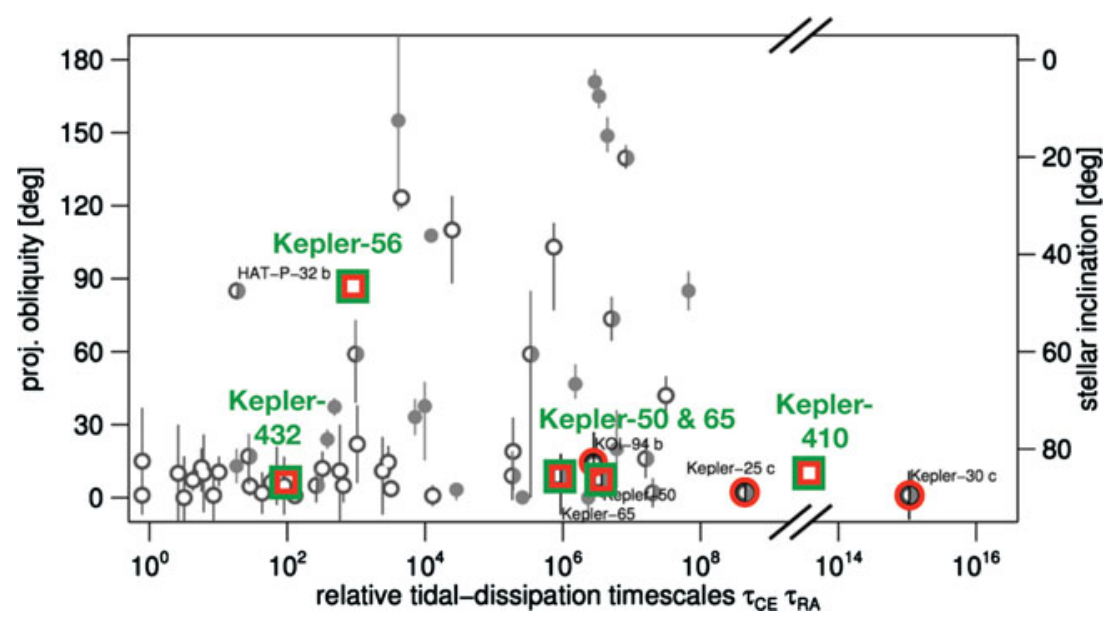

Figure 3. Projected obliquity (left ordinate) and stellar inclination (right ordinate) versus relative tidal dissipation timescales for exoplanet systems. Systems with short dissipation timescales are expected to have been re-aligned even if they were misaligned by the formation process, while systems with long dissipation timescales are expected to preserve their configuration. Multiplanet systems without hot Jupiters are highlighted by red circles, and systems with inclinations measured using asteroseismology are highlighted with green squares. Positions for Kepler-45, Kepler-410 and Kepler-432 are approximate only. Adapted from Albrecht et al. (2013).

\section{Orbital Eccentricities using Asteroseismic Densities}

Exoplanet transits allow a measurement of the semi-major axis relative to the stellar radius $\left(a / R_{*}\right)$, provided the eccentricity of the orbit is known. For circular orbits, $a / R_{*}$ is directly related to the mean density of the star (see, e.g., Seager \& Mallén-Ornelas 2003).

$$
\rho_{\star, \text { transit }}=\frac{3 \pi}{G P^{2}}\left(\frac{a}{R_{\star}}\right)^{3},
$$

where $G$ is the gravitational constant and $P$ is the orbital period. For non-circular orbits, it can be shown that the true stellar density $\left(\rho_{\star}\right)$ is related to the density measured from the transit assuming a circular orbit $\left(\rho_{\star, \text { transit }}\right)$ as (e.g. Kipping 2010):

$$
\frac{\rho_{\star}}{\rho_{\star, \text { transit }}}=\frac{\left(1-e^{2}\right)^{3 / 2}}{(1+e \sin \omega)^{3}}
$$

where $e$ is the orbital eccentricity, and $\omega$ is the argument of periastron. Equations (1) and (2) demonstrate that if an independent measurement of $\rho_{\star}$ is available, transits can be used to directly constrain the eccentricity of a planet without the need for radial velocity observations. Incidentally, a key observable in asteroseismology is the sound travel time through the stellar diameter, which for an ideal gas is directly related to the square root of the mean stellar density (Kjeldsen \& Bedding 1995). Comparisons of asteroseismic densities with densities derived from transiting exoplanets with known eccentricities (Gilliland et al. 2011; Nutzman et al. 2011; Huber 2015) and stellar models (Stello et al. 2009; White et al. 2011) have shown excellent agreement for solar-type stars at the level of a few percent.

The first systematic determination of eccentricities using asteroseismic host star densities was performed by Van Eylen \& Albrecht (2015), using a sample of 28 Kepler multiplanet systems. Figure 4 shows a histogram of the left hand side of equation (2) 


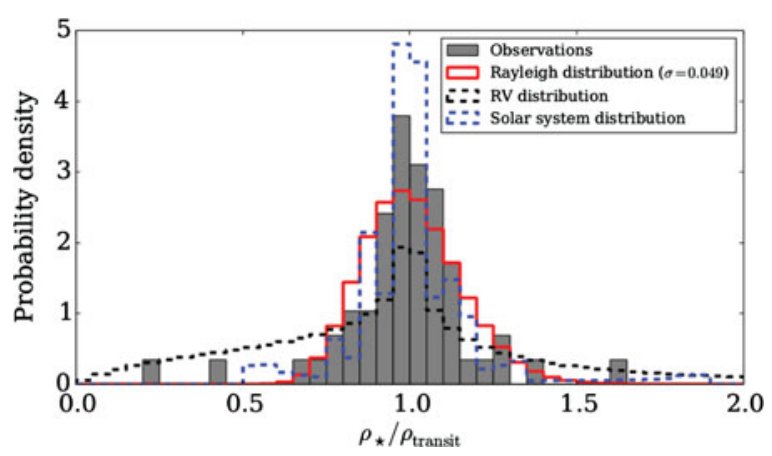

Figure 4. Ratio of the true mean stellar density and the density measured from transits assuming a circular orbit for 28 Kepler multiplanet host stars (grey). Corresponding distributions of solar system planets and planet detected by radial velocity surveys are shown as blue and black dashed histograms, respectively. From Van Eylen \& Albrecht (2015).

for the Kepler sample compared to the solar system as well as a sample of planets with eccentricities determined from radial velocity surveys. The seismic host star sample (red solid lines) is consistent with circular orbits, similar to the solar system (blue dashed histogram) but in stark contrast to the radial velocity sample (black dashed line). Since the seismic host star sample includes mostly small, low-mass planets compared to the more massive planets probed by Doppler surveys, this indicates that low-mass planets are preferentially on circular orbits. This conclusion is of great importance since circular orbits are often explicitly assumed to model the habitable zone (Borucki et al. 2013; Barclay et al. 2013; Quintana et al. 2014; Jenkins et al. 2015) or for estimating transit durations to account for detection completeness in planet occurrence studies (e.g. Howard et al. 2012; Dong \& Zhu 2013; Petigura et al. 2013; Burke et al. 2015). Furthermore, eccentricities are often a key property for constraining planet formation scenarios (such as for hot Jupiters, see previous Section).

\section{Future Prospects}

The repurposed Kepler/K2 Mission is currently conducting 80 day observing campaigns in the ecliptic plane (Howell et al. 2014). While K2 has enabled a large amount of new science, it does not have the ability to follow-up transit detections made with longcadence (30-minute sampling) data with short-cadence (1-minute sampling) observations that are required for asteroseismic analyses of solar-type and subgiant stars. Hence, the only way to study solar-type and subgiant host stars is to either target exoplanet hosts which were known before the K2 campaign started, or revisit already observed fields.

An alternative is to conduct a transit survey for stars which are evolved enough to have oscillation periods detectable with long-cadence data, but small enough to allow the detection of planets. Figure 5 shows the distribution of known planets around evolved $\left(R>3.5 R_{\odot}\right)$ stars in planet mass - orbital period plane. While radial velocity surveys demonstrated that close-in planets with $>5 M_{J}$ are rare around red giants, Kepler has discovered several giant planets orbiting oscillating RGB stars within $\lesssim 0.3 \mathrm{AU}(P \lesssim 50 \mathrm{~d})$ (e.g. Kepler-91b, Kepler-56bc, Kepler432b; Lillo-Box et al. 2014; Huber et al. 2013b; Ciceri et al. 2015). This hints at the existence of a yet poorly studied population of warm Neptune-Saturn mass planets around evolved stars, which has not been probed with radial-velocity surveys. 


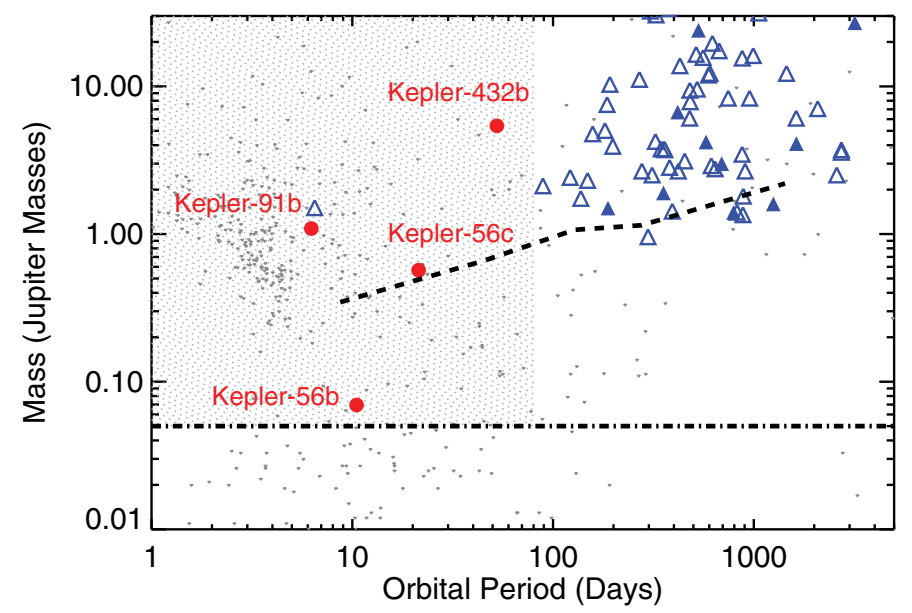

Figure 5. Mass versus orbital period for exoplanets orbiting red-giant branch stars $\left(R>3.5 R_{\odot}\right.$, $T_{\text {eff }}<5500 \mathrm{~K}$ ) detected with transits (red circles) and radial velocities (blue triangles). Open triangles are mean masses assuming random orbit orientations (i.e., $M \sin (i) \pi / 2)$ and grey dots show planets orbiting dwarfs. The dashed line shows the median RV detection limit for mean masses given by Bowler et al. (2010). The dashed-dotted line marks the mass of Neptune as an approximate $\mathrm{K} 2$ detection limit $\left(R \gtrsim 0.5 R_{J}\right)$, and the grey shaded area will be probed by $\mathrm{K} 2$. Note that RV surveys are not sensitive to warm Neptune-Saturn mass planets which will can be detected with $\mathrm{K} 2$.

RGB stars span a wide range of stellar masses, luminosities, and chemical compositions, allowing studies of exoplanets in a variety of host star environments. Both aspects make RGB stars ideal targets to tackle two controversial and unsolved questions in exoplanet science: the occurrence rate of gas-giant planets as a function of stellar mass (e.g., Lloyd 2011; Johnson et al. 2013; Schlaufman \& Winn 2013), and the role of stellar incident flux on the radius inflation of gas-giant planets (Bodenheimer et al. 2001; Chabrier \& Baraffe 2007; Lopez \& Fortney 2015). A K2 transit survey targeting low-luminosity RGB stars can provide important clues to solve these problems.

In addition to K2, future missions such as the Terrestrial Exoplanet Survey Satellite TESS (Ricker et al. 2014) and PLATO (Rauer et al. 2014) will provide opportunities to perform asteroseismology of exoplanet host stars. While the smaller apertures of TESS compared to Kepler will restrict asteroseismic studies to the brightest dwarfs and subgiants, planet yield simulations by Sullivan et al. (2015) indicate $\sim 800$ evolved host stars which may be suited for asteroseismic studies using 30-minute cadence full-frame observations. Additionally, the concept for PLATO includes the capability to detect oscillations of a large number of exoplanet host stars. Combined with ground-based radial-velocity efforts such as the SONG (Grundahl et al. 2008) and LCOGT (Brown et al. 2013) networks, there is little doubt that the synergy between asteroseismology and exoplanet science will continue to thrive over the coming decades.

Acknowledgments: I thank Simon Jeffery and Joyce Guzik for organizing a fantastic IAU focus meeting, as well as Victor Silva Aguirre, Simon Albrecht and Vincent van Eylen for providing figures and comments for this review. Financial support was provided by NASA grant NNX14AB92G and the Australian Research Councils Discovery Projects funding scheme (project number DEI40101364). 


\section{References}

Albrecht, S., Winn, J. N., Marcy, G. W., Howard, A. W., Isaacson, H., \& Johnson, J. A. 2013, ApJ, 771, 11

Albrecht, S., et al. 2012, ApJ, 757, 18

Ballot, J., et al. 2011, A\& A, 530, A97

Barclay, T., et al. 2013, ApJ, 768, 101

Bastien, F. A., Stassun, K. G., Basri, G., \& Pepper, J. 2013, Nature, 500, 427

Batalha, N. M., et al. 2010, ApJ, 713, L109

Bazot, M., Vauclair, S., Bouchy, F., \& Santos, N. C. 2005, A\&SA, 440, 615

Benomar, O., Masuda, K., Shibahashi, H., \& Suto, Y. 2014, PASJ

Bodenheimer, P., Lin, D. N. C., \& Mardling, R. A. 2001, ApJ, 548, 466

Borucki, W. J., et al. 2013, Science, 340, 587

Bouchy, F., Bazot, M., Santos, N. C., Vauclair, S., \& Sosnowska, D. 2005, A $\mathscr{E} A, 440,609$

Bowler, B. P., et al. 2010, ApJ, 709, 396

Brewer, J. M., Fischer, D. A., Basu, S., Valenti, J. A., \& Piskunov, N. 2015, ApJ, 805, 126

Brown, T. M., et al. 2013, PASP, 125, 1031

Buchhave, L. A., et al. 2012, Nature, 486, 375

Burke, C. J., et al. 2015, ApJ, 809, 8

Campante, T. L., et al. 2015, ApJ, 799, 170

Carter, J. A., et al. 2012, Science, 337, 556

Chabrier, G. \& Baraffe, I. 2007, ApJ, 661, L81

Chaplin, W. J. \& Miglio, A. 2013, ARA\& A, 51, 353

Chaplin, W. J., et al. 2013, ApJ, 766, 101

Christensen-Dalsgaard, J., et al. 2010, ApJ, 713, L164

Ciceri, S., Lillo-Box, J., Southworth, J., Mancini, L., Henning, T., \& Barrado, D. 2015, A\&̋A, $573, \mathrm{~L} 5$

Dong, S. \& Zhu, Z. 2013, ApJ, 778, 53

Gilliland, R. L., McCullough, P. R., Nelan, E. P., Brown, T. M., Charbonneau, D., Nutzman, P., Christensen-Dalsgaard, J., \& Kjeldsen, H. 2011, ApJ, 726, 2

Gizon, L. \& Solanki, S. K. 2003, ApJ, 589, 1009

Gizon, L., et al. 2013, Proceedings of the National Academy of Science, 110, 13267

Grundahl, F., Christensen-Dalsgaard, J., Kjeldsen, H., Frandsen, S., Arentoft, T., Kjaergaard, P., \& Jørgensen, U. G. 2008, in IAU Symposium, Vol. 252, IAU Symposium, ed. L. Deng \& K. L. Chan, 465-466

Howard, A. W., et al. 2012, ApJS, 201, 15

Howell, S. B., Everett, M. E., Sherry, W., Horch, E., \& Ciardi, D. R. 2011, AJ, 142, 19

Howell, S. B., et al. 2014, PASP, 126, 398

Huber, D. 2015, in Astrophysics and Space Science Library, Vol. 408, Giants of Eclipse: The zeta Aurigae Stars and Other Binary Systems, 169

Huber, D., et al. 2013a, ApJ, 767, 127

Huber, D., et al. 2013b, Science, 342, 331

Jenkins, J. M., et al. 2015, AJ, 150, 56

Johnson, J. A., Morton, T. D., \& Wright, J. T. 2013, ApJ, 763, 53

Kipping, D. M. 2010, MNRAS, 407, 301

Kjeldsen, H. \& Bedding, T. R. 1995, A\&A, 293, 87

Lebreton, Y. \& Goupil, M. J. 2014, $A \& A$, 569, A21

Lillo-Box, J., et al. 2014, $A \mathscr{E} A, 568, \mathrm{~L} 1$

Lissauer, J. J., et al. 2011, Nature, 470, 53

Lloyd, J. P. 2011, ApJ, 739, L49

Lopez, E. D. \& Fortney, J. J. 2015, ApJ, in press (arXiv:1510.00067)

Lund, M. N., et al. 2014, A\&A, 570, A54

Metcalfe, T. S., et al. 2012, ApJ, 748, L10

Mullally, F., et al. 2015, ApJS, 217, 31

Nutzman, P., et al. 2011, ApJ, 726, 3 
Petigura, E. A., Howard, A. W., \& Marcy, G. W. 2013, PNAS, 110, 19175

Quinn, S. N., et al. 2015, ApJ, 803, 49

Quintana, E. V., et al. 2014, Science, 344, 277

Rauer, H., et al. 2014, Experimental Astronomy

Ricker, G. R., et al. 2014, in Society of Photo-Optical Instrumentation Engineers (SPIE) Conference Series, Vol. 9143, Society of Photo-Optical Instrumentation Engineers (SPIE) Conference Series, 20

Sanchis-Ojeda, R., et al. 2013, ApJ, 775, 54

Schlaufman, K. C. \& Winn, J. N. 2013, ApJ, 772, 143

Seager, S. \& Mallén-Ornelas, G. 2003, ApJ, 585, 1038

Silva Aguirre, V., et al. 2015, MNRAS, 452, 2127

Steffen, J. H., et al. 2012, MNRAS, 421, 2342

Stello, D., et al. 2009, ApJ, 700, 1589

Sullivan, P. W., et al. 2015, ApJ, 809, 77

Van Eylen, V. \& Albrecht, S. 2015, ApJ, 808, 126

Van Eylen, V., et al. 2014, ApJ, 782, 14

White, T. R., Bedding, T. R., Stello, D., Christensen-Dalsgaard, J., Huber, D., \& Kjeldsen, H. 2011, ApJ, 743, 161

Winn, J. N., Fabrycky, D., Albrecht, S., \& Johnson, J. A. 2010, ApJ, 718, L145 